\title{
Methods for Calculating Activation Energies of Ideal
}

\section{Gases}

\author{
Richard Martin Gibbons \\ 4 Little Acre, Beckenham, Kent BR3 3ST, UK
}

\begin{abstract}
The previous paper Ref. [1] showed how to calculate activation energies for ideal gas reactions from the CDF (cumulative distribution function) of the MBD (Maxwell Boltzmann Distribution) and the heat capacity data of the components. The results presented here show comparisons of activation energies of four ideal gases calculated in that way with those calculated from the ND (Normal Distribution) and its CDF. The evaluation of the CDF for the MBD in Ref. [1] required extensive numerical integrations for each substance. In this paper this method of calculating activation energies is generalised, by showing the CDF is a unique function, independent of temperature and composition, enabling the CDF to be presented graphically or in tabular form. These activation energies are compared to those calculated from the ND and its CDF. The MBD is related to the ND because it has a generating function which is shown here to have the simple form $(1-\mathrm{k} T)^{-1.5}$. The activation energies obtained from the CDF of the ND are shown to agree ca. $5-7 \%$ with those obtained directly from the MBD. Because existing thermodynamic treatments are based on average properties, they cannot give either a complete account of thermodynamic controlled and kinetic controlled equilibrium states or explain transitions between them. Complete treatments must include effects from the MBD which are the causes of kinetic controlled equilibrium. The basis for a complete treatment is outlined, which includes the standard deviations and activation energies.
\end{abstract}

Key words: Thermodynamics, statistical mechanics, equilibrium, reactions of ideal gases.

\section{Introduction}

The first paper of this series [1] showed how to calculate activation energies for ideal gas reactions from the MBD (Maxwell Boltzmann Distribution), its cumulative distribution function, CDF (cumulative distribution function), and the heat capacities of the components. The results showed that the collision kinetic energies alone are too small, compared to bond energies, to provide mechanisms for these reactions. Nor can they explain why ignition temperatures, $T_{\mathrm{ign}}$, are well defined. The lack of correlations for the heat capacities of the components that include excitation of electrons is the main reason that activations energies cannot be calculated accurately. These issues show the need for accurate correlations for ideal gas heat capacities that include excitations of electrons to

Corresponding author: Richard Martin Gibbons, Ph.D., DIC retired, research fields: thermodynamics, statistical mechanics and thermo-physical properties. calculate accurate activation energies.

Those calculations of activation energies were based on the CDF for the MBD of the components involved in the reactions. How this approach can be generalised in a way that avoids numerical integrations is discussed below. But first an alternative treatment that also avoids numerical integrations is discussed. It is based on the ND and its CDF, for which there are widely available tables of values which obviate the need to do numerical integrations. It involves approximating the MBD by an ND and was the first method the author used to calculate activation energies. It was to resolve questions about how accurately the ND approximated the MBD that led to the method discussed in Ref. [1].

To show the MBD is related to the ND it is necessary to demonstrate it has a generating function and it is shown here that the MBD has the simple form $(1-\mathrm{k} T)^{-1.5}$. Hence the two distributions are mathematically related and the CDF for the ND can be 
used in calculating energy distributions in the MBD.

Activation energies obtained for four gas ideal mixtures from the CDF of the ND agree to ca. $5-7 \%$ with those obtained directly from the MBD.

\section{The Energy Distribution for the MBD}

Quantitative calculations of the energy distribution in the MBD require knowledge of the mole fraction $x_{\mathrm{i}}$ with energies greater than a specific energy $u_{\mathrm{i}}$. The standard expression for the MBD is from Ref. [2]:

$$
p(u)=A e^{\left(-\frac{u}{k T}\right)} c^{2}
$$

where $c$ is the velocity, $m$ is the mass, the temperature $T$ and k is Boltzmann's constant and,

$$
A=4 \pi\left(m /(2 \pi k T)^{1.5}\right.
$$

To obtain the mole fraction, $y_{\mathrm{i}}$, with $u<u_{\mathrm{i}}$ this equation must be integrated between 0 and $c_{i}$.

$$
y_{i}=A \int_{0}^{c_{i}} e^{(-u / k T)} c^{2} d c
$$

These integrals are intractable and cannot be solved analytically. As discussed in Ref. [1] they are simply and accurately evaluated numerically by summing the expression for $x_{\mathrm{i}}$ in equation1 using an appropriate grid spacing for the values of $c$.

An alternative method of calculating activation energies is to relate MBD to the ND and use the CDF for the ND to calculate the activation energies. How to do this is discussed below.

\section{The MBD and the Normal Distribution}

Any distribution that has an MGF (moment generating function) [3] is related to the ND.

To relate MBD to the ND it is sufficient to show it has an MGF. This enables the tables for the CDF of the ND to be used for calculating mole fractions of molecules with energies $>u_{\mathrm{i}} / u_{\mathrm{av}}$.

The moments of the MBD, $M_{n}(u)$ are defined in Eq. (4) as,

$$
M_{n}(u)=\int_{0} A e^{\left(-\frac{u}{k T}\right)} u^{n} c^{2} d c
$$

Moments can be evaluated directly from this equation and the first four moments are tabulated in Table 1 . The standard procedure in statistics to obtain a generating function is to form the function:

$$
M(t)=\int A e^{t 0.5 m c^{2}} e^{-a c^{2}} c^{2} d c
$$

On carrying out the integration

$$
M(t)=(1-k T)^{-1.5}
$$

On expansion of this function the coefficients of the powers of $t$ are the moments of the MBD as can be seen by comparing those coefficients with the direct evaluations of the first four moments in Table 1.

The variable in the MBD that is related to the ND is the velocity, $c$, not the energy, $u$.

To relate the MBD to the ND it is necessary to transform it into a normalised form [4] using a standard transformation either about zero or the average energy, $u_{\mathrm{av}}$. The expressions for the expansion about zero lead to much simpler algebraic forms, for the normalised MBD and are used below. The normalised form for a variable $x, x^{*}$, is

$$
x^{*}=x / \sigma
$$

where $x^{*}$ is the normalised value of $x, \sigma$ is the standard deviation and $x_{\mathrm{av}}$ is the average value of $x$. The normalised values of $c$ and $u$ are:

$$
\begin{gathered}
\sigma c^{*}=c \\
u^{*}=\frac{u}{\sigma^{2}}
\end{gathered}
$$

For ideal gases $0.5 m \sigma^{2}$ is equal to $1.5 \mathrm{k} T$ so the parameter $\mathrm{u} / \mathrm{kT}$ becomes

$$
u^{*} / k T=b u /(b k T)=\frac{b u}{\sigma^{2}}
$$

And $b$ is $(3 / 2)$. The derivatives $\mathrm{d} c^{*}$ and $\mathrm{d} c$ are related

$$
\sigma d c^{*}=d c
$$

Table 1 The moments of the MBD.

\begin{tabular}{lll}
\hline$n$ & $M_{n}(0)$ & $M_{n}(1.5 \mathrm{k} T)$ \\
1 & $1.5 \mathrm{k} T$ & $1.5 \mathrm{k} T$ \\
2 & $15 / 4(\mathrm{k} T)^{2}$ & $6 / 4(\mathrm{k} T)^{2}$ \\
3 & $105 / 8(\mathrm{k} T)^{3}$ & $3(\mathrm{k} T)^{3}$ \\
4 & $945 / 16(\mathrm{k} T)^{4}$ & $252 / 16(\mathrm{k} T)^{4}$ \\
\hline & \multicolumn{3}{l}{ Calculated directly from the generating function: about zero } \\
and about the average kinetic energy.
\end{tabular}


Restating Eq. (3) in terms of $c^{*}$ and $u^{*}$ about zero, it becomes:

$$
x_{c^{*}}=A b^{3} \int_{0}^{c^{*}} e^{\left(-b u^{*} / \sigma^{2}\right)} c^{*^{2}} d c^{*}
$$

This expression for $x_{\mathrm{c}}{ }^{*}$ is related to the ND for the ratio $c^{2} / c_{\mathrm{av}}{ }^{2}$. Multiplying each of these squared velocities by $0.5 \mathrm{~m}$ converts this ratio into $u / u_{\mathrm{av}}$.

The percentage of molecules needed for the reaction to proceed, $y_{\mathrm{ign}}$, is obtained, as previously in Ref. [1], using experimental values for the reactions of ideal gases. This leads to the same equation as in Ref. [1] where $x_{\mathrm{i}}$ is the mole fraction of $\mathrm{i}$ and $\mathrm{i}$ is $\mathrm{H}_{2}$, $\mathrm{O}_{2}$ or $\mathrm{N}_{2}$. The heat of reaction is $\Delta H$ at standard conditions.

For the mixture to reach the ignition temperature the heat released by the reaction of the mole fraction of hydrogen, $y_{\text {ign }}$, leads to:

$$
\begin{gathered}
y_{i g n} \Delta H=\int_{T_{o}}^{T_{i g n}}\left(x_{H_{2}} C_{p_{H_{2}}}+x_{N_{2}} C_{p_{N_{2}}}+\right. \\
\left.x_{O_{2}} C_{p_{O_{2}}}\right) d T
\end{gathered}
$$

and the $x$ values are the mole fractions, $C_{\mathrm{p}}$ is the heat capacity at constant pressure, $T_{\mathrm{o}}$ is $25{ }^{\circ} \mathrm{C}$ and the subscripts identify the components. Alternatively, this equation can be written in terms of the enthalpies of the components:

$$
y_{i g n} \Delta H_{R}=x_{H_{2}} \Delta H_{p_{H_{2}}}+x_{N_{2}} \Delta H_{p_{N_{2}}}+x_{O_{2}} \Delta H_{p_{O_{2}}}(14)
$$
where $\Delta H_{\mathrm{R}}$ is the heat of reaction and the $\Delta H_{\mathrm{x}}$ values are the enthalpy differences between $T_{\text {ign }}$ and $T_{0}$ for each component.

The quantities in this equation were obtained using the correlations of Harmens [5] to evaluate the heat capacities of the components together with the reaction enthalpies in the same way as in Ref. [1]. The results are discussed below.

\section{Activation Energies of Ideal Gas Reactions}

Activations energies and associated data are shown in Table 2 for an ND about the average energy. The mole fractions, $y_{\mathrm{ign}}$, in Eqs. (1) or (2) are the ignition mole fractions of the fuel gases which, when combusted successfully, heat an equal number of molecules in the mixture of fuel and air to the $T_{\text {ign }}$, ensuring that the combustion is sustained. They contain by definition those molecules with sufficient energy to overcome the activation energy barriers which otherwise prevent molecules from reacting. As shown in Ref. [1] their energies are at least equal to those of the activation energy barriers. Comparisons with the bond energies in Table 3 lead to the same conclusion as in Ref. [1] that the kinetic energies of collision occur as collision energies which are always $<20 \%$ of the bond energies but nevertheless disrupt bonds energies five times larger.

Activation energies calculated from the MBD and ND about the average zero are compared with those calculated from the MBD and its CDF in Table 2 where it can be seen there are differences of ca. 5-7\%. These differences are unexplained but too small to affect any of the conclusions that arise from these results. In part they may be due to the fact that MBD is not accurately approximated by the ND. This conjecture is supported by the results of calculations of $U_{\text {act }}$ from the ND about zero and $U_{\text {av }}$ which differ from one another by about 5\%. By contrast calculations based on the MBD and its CDF provide a rigorous root to activation energies, once accurate heat capacity correlations and data for the CDF of the MBD are available.

Table 2 Combustion data for four fuels.

\begin{tabular}{lllllll}
\hline Fuel & $\begin{array}{l}x_{\mathrm{f}}[6] \\
\text { flammability } \\
\text { limit }\end{array}$ & $T_{\mathrm{ign}} / \mathrm{K}[6]$ & $\begin{array}{l}y_{\text {ign }} \\
\text { mole fraction }\end{array}$ & $U_{\mathrm{ign}} / U_{\mathrm{av}}$ from ND $\begin{array}{l}\text { Collision energy } \\
\text { at } T_{\mathrm{ign}}(\mathrm{kJ} / \mathrm{mol})\end{array}$ & $\begin{array}{l}U_{\mathrm{ign}} / U_{\mathrm{av}} \\
\text { from MBD [1] }\end{array}$ \\
\hline Hydrogen & 0.040 & 847 & 0.0290 & 3.0 & 61.3 & 2.9 \\
Methane & 0.050 & 905 & 0.0360 & 2.85 & 63.2 & 2.8 \\
Ethane & 0.031 & 745 & 0.0089 & 3.9 & 63.3 & 3.4 \\
Propane & 0.021 & 754 & 0.0057 & 4.1 & 66.6 & 3.5 \\
\hline
\end{tabular}


Table 3 Bond energies and kinetic energies at ignition temperatures.

\begin{tabular}{llll}
\hline Fuel & Bond energy $(\mathrm{kJ} / \mathrm{mol})$ & $\begin{array}{l}\Delta H \text { Bond energy }[7] \\
(\mathrm{kJ} / \mathrm{mol})\end{array}$ & Collision energies $(\mathrm{kJ} / \mathrm{mol})$ \\
\hline Hydrogen & $\mathrm{H}-\mathrm{H}$ & 436 & 61.3 \\
Methane & $\mathrm{C}-\mathrm{H}$ & 412 & 63.2 \\
& $\mathrm{C}-\mathrm{C}$ & 348 & \\
Ethane & $\mathrm{C}-\mathrm{H}$ & 412 & 63.4 \\
& $\mathrm{C}-\mathrm{C}$ & 348 & 66.6 \\
Propane & $\mathrm{C}-\mathrm{H}$ & 412 & \\
& $\mathrm{C}-\mathrm{C}$ & 348 & \\
\hline
\end{tabular}

\section{Simplified Calculation Methods for Activation Energies}

Prior to the results in Ref. [1], calculation of activation energies had to involve the ND and its CDF. The properties of the CDF for the MBD suggest a simpler way of obtaining activation energies using the interpolated values of $x$ and $u / u_{\mathrm{av}}$ listed in Table 1 of Ref. [1]; for convenience these values are listed in Table 4.

The Boltzmann factor is dimensionless and consequently the distributions of kinetic energies are independent of temperature while the averages do depend on temperature. Introducing the parameter, $c_{0}$, the average molecular velocity, it immediately follows that the MBD can be written in terms of $c_{i}{ }^{*}$, defined as the ratio $c_{\mathrm{i}} / c_{0}$. Noting that

$$
0.5 m c_{0}^{2}=1.5 k T
$$

The MBD becomes in terms of $c^{*}$

$$
x_{i}=\int_{0}^{c_{i}} A e^{-1.5 c^{* 2}} c^{* 2} c_{0}^{2} d c
$$

Writing this equation in terms of $x_{\mathrm{i}} / c_{0}{ }^{2}$ and $c^{*}$ [2] shows there is a single expression for the kinetic energies of the MBD that applies to all substances at all temperatures.

This is confirmed by the calculated values of the mole fractions for hydrogen for selected values of the molecular velocities and $u / u_{\mathrm{a}}$, at its ignition temperature, $500 \mathrm{~K}$ and at $298.15 \mathrm{~K}$, only the data for $298.15 \mathrm{~K}$ are shown in Fig. 1. The plots of values of $x$ and $u / u_{\mathrm{a}}$ are identical at all three temperatures. Identical results were obtained for the other gases at each value of the ratios of $c^{2} / c_{0}^{2}$ at both $298.15^{\circ} \mathrm{C}$ and their ignition temperature. This is further confirmed by the data for $y_{\mathrm{ign}}$ for all four gases fall on a single plot of $x$ versus $u / u_{\mathrm{a}}$ for hydrogen, even though the velocities of the individual gases differ by the square root of the ratio of their molecular weights; this is a factor-four for hydrogen and propane.

These considerations implied there is a generic function that applies to all kinetic energies at all temperatures. The expression in Eq. (16) can be reformulated using Eq. (15), to replace $\mathrm{k} T$ by $0.5 m c_{0}^{2}$. When inserted into Eq. (16) these lead to the following expressions:

Table 4 Values of $x$ and $u / u_{\mathrm{a}}$ for the MBD.

\begin{tabular}{llll}
\hline $\begin{array}{l}\text { Mole fraction } \\
x>u / u_{\mathrm{av}}\end{array}$ & $u / u_{\mathrm{a}}$ & $\begin{array}{l}\text { Interpolated } \\
\text { mole fractions }\end{array}$ & $\begin{array}{l}\text { Interpolated } \\
u / u_{\mathrm{a}}\end{array}$ \\
\hline 0.0505 & 2.600 & 0.005 & 4.3 \\
0.0012 & 2.750 & 0.010 & 3.8 \\
0.03348 & 2.905 & 0.020 & 3.5 \\
0.02693 & 3.0064 & 0.025 & 3.10 \\
0.01712 & 3.395 & 0.03 & 3.00 \\
0.01351 & 3.569 & & \\
0.0106 & 3.740 & & \\
0.0049 & 4.297 & & \\
\hline
\end{tabular}




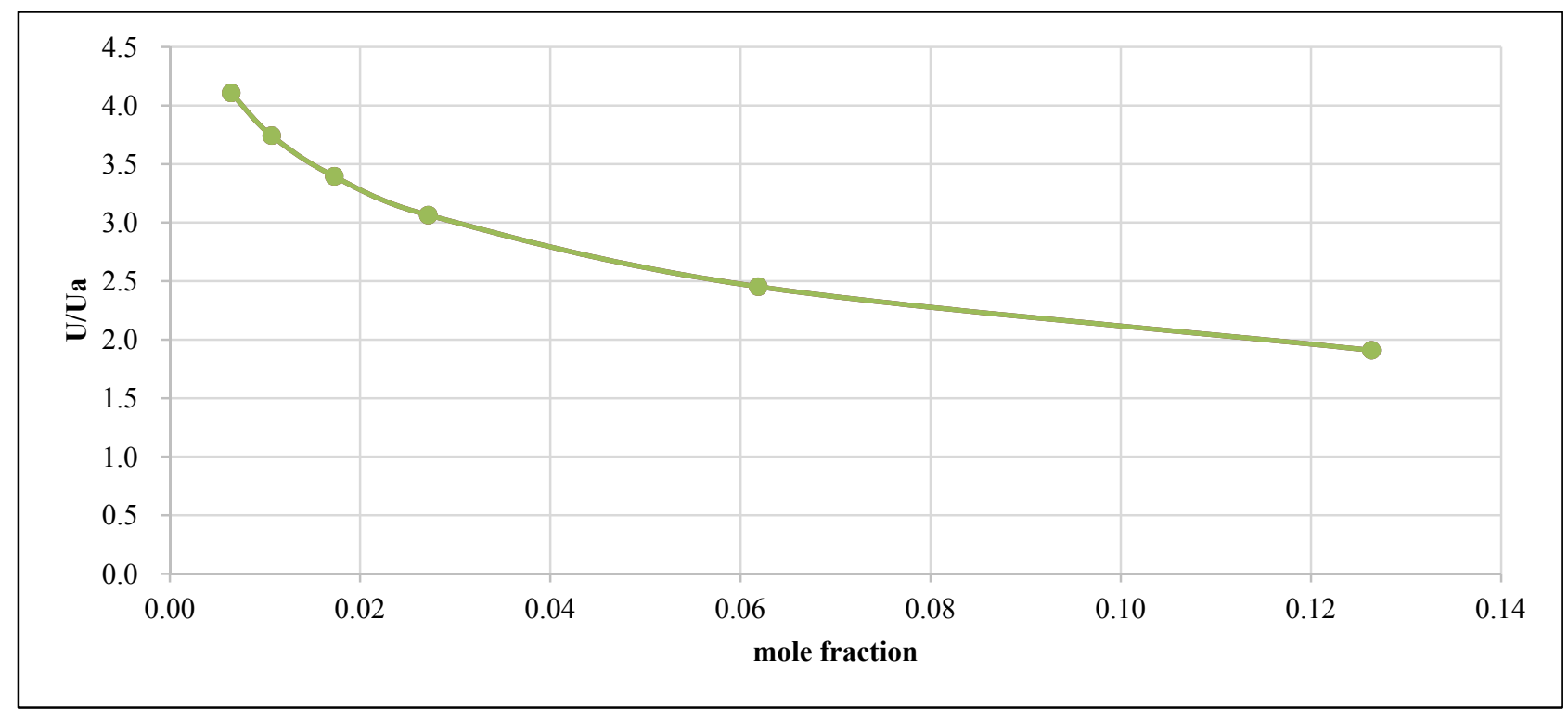

Fig. 1 Activation energies for hydrogen at 298.15 K.

$$
\begin{gathered}
p\left(c^{*}\right)=A_{M B} e^{-1.5 c^{*^{2}}} c^{*^{2}} \\
A_{M B}=6 /(1.5 \pi)^{0.5}
\end{gathered}
$$

where $p\left(c^{*}\right)$ is the probability that the value of $c^{*}$ occurs and the value of $A_{\mathrm{MB}}$ ensures that the probabilities are normalised. The expression for $p\left(c^{*}\right)$ is closely related to the normal distribution.

The interpolated values for $u / u_{\mathrm{a}}$ in Table 4 enable the activation energy to be read from the graph, once $y_{\text {ign }}$ has been obtained. The values from the graph are probably accurate enough for most purposes. Alternatively, a closely spaced table of values for the $\mathrm{CDF}$ of the MBD for the range important to calculating activation energies, of $2.0<u / u_{a}<4.5$, is readily generated from Eqs. (17) and (18).

\section{Discussion}

The values for the activation energies in this paper [1] are of interest in themselves in that they are the first attempts to calculate activation energies. The basic data in the form of accurate heat capacity are not available for these calculations to be accurate are unfortunate. But these results do show that the existing explanation of reactions based on kinetic energies of collisions is not sustainable and identify the need for accurate heat capacity data at ignition temperatures to enable activation energies to be calculated accurately.

It is clear that, for equilibrium states determined by the distribution of energies in the MBD, a full explanation of those states must account for effects caused by the distribution of energies. Explanations based solely on averages of the properties of the molecules cannot account for these effects.

Perhaps more important is the demonstration of incompleteness of thermodynamic treatments that involve kinetic controlled equilibrium. Such kinetic controlled equilibriums are metastable states. Standard treatments do not explain why these states exist nor can they deal with the transition to thermodynamic controlled equilibrium. The criteria for the propagation of combustion reactions set out in these two articles are the first criteria for transitions between kinetic and thermodynamic controlled equilibrium.

The cause of this problem is that kinetic controlled equilibrium states arise from the distributions of molecular energies and standard treatments are based on properties that are averages. They contain no information about the distributions of molecular energies. All molecular systems have an MBD of energies and this must be included in thermodynamic treatments that can describe quantitatively both 
thermodynamic and kinetic controlled equilibrium and transitions between these two types of equilibrium. Any such treatment will be based on the average properties, as is in current treatments, the standard deviations of the molecular energies, to account for the distribution those energies, and activation energies.

Activation energies of ideal gas reactions have an odd status in thermodynamic theory. They control reactions and are determined by the equilibrium molecular energy distributions and the criteria for propagation of the reactions. Together with the criteria for propagation they determine what equilibrium will occur. They are the only parameters which determine thermodynamic equilibrium states which are not recognised as a thermodynamic property. Currently, there are no ways of calculating them accurately accounts no doubt accounts for this.

\section{Conclusions}

Statistical distributions play a central role in the behaviour of molecular systems and in thermodynamic equilibrium. They determine how thermodynamic controlled and kinetic controlled equilibriums are related. Chemical theories describing molecular systems must include the effects of energy distributions as they play an essential role in kinetic controlled equilibriums.

This paper and Ref. [1] give two methods for calculating activation energies for the reactions of ideal gases. The method based on the MBD and its
$\mathrm{CDF}$ is the most rigorous and has no additional assumptions. The simplest way to use it, once $y_{\text {ign }}$ has been calculated, is to read the activation energy from Fig. 1.

The values obtained from the MBD and the ND contain additional assumptions and differ by ca. $5-7 \%$ from those obtained from the MBD and its CDF. These differences are probably due to errors in the heat capacity data leading to errors in the $y_{\text {ign }}$ values rather than differences in the mathematical procedures. The real advantage of the linking the MBD and the ND mathematically is that it relates the MBD to the central limit theorem. These links provide the basis for a simple introduction to statistics and thermodynamics for all students of chemistry.

\section{References}

[1] Gibbons, R. M. 2016. "Thermodynamic Equilibrium." Journal of Energy and Power Engineering 10 (10): 623-7.

[2] Roberts, J. K. 1956. Heat and Thermodynamics (4th ed.). London: Blackie and Son, 80-101.

[3] Hugill, M. 1985. Advanced Statistics. Vancouver: Collins Educational, ISBN: 0-00-322215-2, 400-18.

[4] Attwood, G., and Skipworth, G. 2001. Statistics 1. Portsmouth, NH: Heinemann Educational Publishers, ISBN 0435518119, 198-201.

[5] Harmens, A. 1979. Fluids and Fluids Mixtures. NPL ISBN: 086030095, 112-21.

[6] Perry, J. 1953. Chemical Engineers Handbook (2nd ed.). New York, NY: McGraw Hill, 1582-86.

[7] Stack, J. G., and Wallace, H. G. 1970. Chemistry Data Book. London: John Murray, ISBN: 0-7195-3951-X, 32-54. 